\title{
Aversão alimentar adquirida e qualidade de vida em mulheres com neoplasia mamária ${ }^{1}$
}

\author{
Acquired food aversion and quality of life \\ in women with beast cancer
}

Bruna Mara Okano SÃO PEDRO²

Mário MOURÃO NETTO3

Nágila Raquel Teixeira DAMASCENO2

\section{RE S U M O}

\section{Objetivo}

Avaliar o comportamento alimentar de mulheres com câncer de mama submetidas à quimioterapia, e sua relação com a qualidade de vida destas pacientes.

\section{Métodos}

A partir de um ensaio clínico do tipo antes e depois, selecionou-se 25 mulheres do Hospital AC Camargo (São Paulo, Brasil) durante o período de outubro de 2005 a abril de 2006. As pacientes inclusas no estudo apresentavam diagnóstico de câncer de mama, com estadiamento I e ll e indicação de tratamento quimioterápico adjuvante. Nos momentos T0 (antes) e T1 (após o tratamento quimioterápico), o comportamento alimentar (consumo e aversão alimentar) foi avaliado por três recordatórios 24 horas e um questionário Food Action, respectivamente. A qualidade de vida foi monitorada por meio do questionário Functional Assessment of Cancer Therapy-Breast.

\section{Resultados}

Após o tratamento quimioterápico $(\mathrm{T} 1)$, o consumo de macro e micronutrientes não apresentou alterações significantes, mas o consumo de frutas e sucos aumentou $(p=0,03)$. Perfil inverso foi observado em relação à preferência por café preto $(p=0,01)$ e pelo grupo de bebidas $(p<0,001)$. Alimentos gordurosos $(38 \%)$, laticínios $(23 \%)$, café preto $(15 \%)$, chá $(15 \%)$, chocolate $(7 \%)$ e carne vermelha $(7 \%)$ foram os principais alimentos associados ao desconforto das pacientes. Análises de qualidade de vida mostraram que o tratamento

1 Artigo elaborado a partir da dissertação de S.M.M.L. VERDE, intitulada "Impacto do tratamento quimioterápico no estado nutricional e no comportamento alimentar de pacientes com neoplasia mamária e suas consequências na qualidade de vida". Faculdade de Saúde Pública; 2007. Apoio: Fundação de Amparo à Pesquisa do Estado de São Paulo (Processo 05/50457-0), Conselho Nacional de Pesquisa (Processo 135470/2005-6) e Pró-Reitoria de Pesquisa da Universidade de São Paulo (Projeto 4, PRP/USP/2006 $1^{\circ}$ semestre/057).

2 Universidade de São Paulo, Faculdade de Saúde Pública, Departamento de Nutrição. Av. Dr. Arnaldo, 715, Cerqueira César, 01246-704, São Paulo, SP, Brasil. Correspondência para/Correspondence to: N.R.T. Damasceno. E-mail: <nagila@usp.br>.

3 Fundação Antônio Prudente, Hospital AC Camargo, Departamento de Mastologia. São Paulo, SP, Brasil. 
796 | S.M.M.L. VERDE et al.

quimioterápico promoveu significante redução no bem estar físico $(p<0,01)$. Após o mesmo, algumas variáveis do comportamento alimentar foram significantemente correlacionadas com os parâmetros de qualidade de vida.

\section{Conclusão}

A relação bilateral entre comportamento alimentar e qualidade de vida foi modificada negativamente pelo tratamento quimioterápico.

Termos de indexação: Câncer de mama. Comportamento alimentar. Qualidade de vida. Quimioterapia.

\section{A B S T R A C T}

\section{Objective}

The objective of this paper was to assess the eating behavior of women with breast cancer subjected to chemotherapy and its relation with the quality of life of these patients.

\section{Methods}

A total of 25 women receiving care at the Hospital AC Camargo (SP, Brazil) from October 2005 to April 2006 were selected based on a clinical assay of the before and after type. The patients included in the study were diagnosed with breast cancer, stages I and II and had indication of adjuvant chemotherapy. At the times TO (before) and T1 (after chemotherapy), the eating behavior (food intake and aversion) was assessed by three 24-hour recalls and the Food Action questionnaire, respectively. Quality of life was monitored through the use of the Functional Assessment of Cancer Therapy - Breast questionnaire.

\section{Results}

After chemotherapy (T1), the intake of macro and micronutrients did not change significantly, but the intake of fruits and juices increased ( $p=0.03)$. The opposite was observed regarding the preference for black coffee $(p=0.01)$ and for the beverages group $(p<0.001)$. Fat-rich foods $(38 \%)$, dairy $(23 \%)$, black coffee $(15 \%)$, tea $(15 \%)$, chocolate $(7 \%)$ and red meats (7\%) were the main foods associated with patient discomfort. Analyses of the quality of life showed that chemotherapy caused a significant reduction of physical well-being $(p<0.01)$. After chemotherapy, some eating behavior variables were significantly correlated with quality of life parameters.

\section{Conclusion}

Chemotherapy caused the bilateral relationship between eating behavior and quality of life to change in a negative manner.

Indexing terms: Breast neoplasms. Feeding behavior. Quality of life. Drug therapy.

\section{N T R O D U ÇÃ O}

O câncer de mama é a principal causa de morte entre as mulheres, e o segundo tipo de câncer que mais atinge a população brasileira. 0 diagnóstico dessa neoplasia traz consigo um estigma negativo, independentemente do prognóstico da doença ${ }^{1}$.

O registro de prevalência do câncer de mama no mundo é de quase quatro milhões de pacientes ${ }^{2}$ os quais, apesar do sucesso efetivo do tratamento antineoplásico, passam a conviver com outros problemas, tais como: 1) impacto psicossocial do tratamento; 2) as chances de recidiva da doença e; 3) a associação a outras comorbidades (doenças cardiovasculares, diabetes, osteoporose $)^{3,4}$.

Segundo o Instituto Nacional do Câncer ${ }^{5}$, a estimativa de novos casos de câncer de mama para o ano de 2008 foi de quase 50 mil, sendo mais de 15 mil no Estado de São Paulo. De acordo com Pinho \& Coutinho ${ }^{6}$, de 1980 a 2000, a mortalidade por câncer de mama aumentou de 6,14 para 9,64 por 100 mil mulheres, resultando em um aumento relativo de $57 \%$ e, portanto, representando um dos maiores problemas de saúde pública para o País.

A quimioterapia, de forma geral, promove o aparecimento de sérios efeitos colaterais em 
mulheres com neoplasia mamária. Fadiga, náuseas e vômitos, alterações na função intestinal e alteração na percepção do paladar, são problemas comuns?.

Em conseqüência, essas pacientes podem mostrar alteração do comportamento alimentar devido a uma série desses efeitos, apresentando diminuição da ingestão induzida pela presença de náuseas, alteração do paladar e/ou olfato, inadequação do consumo em função da dor causada pelas mucosites ${ }^{8}$. Dessa forma, torna-se difícil manter a alimentação habitual ${ }^{9}$.

Os protocolos quimioterápicos disponíveis estão associados ao desenvolvimento de aversões alimentares, que resultam da associação entre o mal-estar promovido pelo tratamento e o alimento simultaneamente consumido ${ }^{10,11}$. Por meio da menor diversidade dos alimentos consumidos, a aversão alimentar adquirida pode exercer um papel prejudicial tanto no estado nutricional, quanto na qualidade de vida das pacientes com câncer ${ }^{11}$.

O típico sabor das refeições constitui uma das poucas constantes na vida e é, geralmente, um item seguro no qual se pode procurar conforto. Entretanto, alterações no sabor de alimentos já conhecidos podem ter um impacto bastante pronunciado, particularmente quando ocorrem em um período emocionalmente negativo, como após o diagnóstico ou durante o tratamento de câncer. Por essa razão, mudanças na relação das pacientes com o alimento podem influenciar sua resposta ao tratamento, à medida que comprometem a disposição da paciente e possibilitam o surgimento de um quadro depressivo, o que, por sua vez, debilita a qualidade de vida pelo fato de os alimentos preferidos e altamente consumidos serem os maiores alvos dessa rejeição, diminuindo assim as chances de sobrevivência 8,11, 12 .

Ademais, essas aversões podem implicar em perda de apetite e em conseqüente modificação do peso, o que pode compromete o estado nutricional da paciente e, assim, sua resposta ao tratamento ${ }^{13}$.

A eficácia dos tratamentos disponíveis não é mais mensurada exclusivamente pelas taxas de mortalidade, mas sim pela qualidade de vida que proporciona aos pacientes, a qual pode ser definida como conceito subjetivo, pessoal e multidimensional ${ }^{14,15}$. A mensuração da qualidade de vida de um paciente pode, dessa forma, ser utilizada na prática clínica para a tomada de decisões ${ }^{15}$. Nesse contexto, fornecer informações acuradas, realistas e compreensíveis sobre o tratamento, os efeitos colaterais e os cuidados de saúde necessários é um fator essencial à capacitação e ao reforço das habilidades das pacientes em lidar com sua situação atual e na melhora de sua qualidade de vida ${ }^{7}$.

Reconhecer estes problemas e seu impacto real permite aos profissionais de saúde fornecer informações mais completas sobre eles, as quais capacitarão os pacientes a escolher e lidar melhor com a forma de tratamento antineoplásico a ser administrada.

Nesse sentido, o presente estudo teve como objetivos avaliar o impacto do tratamento quimioterápico na aquisição de aversões alimentares em mulheres com neoplasia mamária, e investigar se uma possível alteração no comportamento alimentar pode ter conseqüências diretas na qualidade de vida dessas pacientes.

\section{M É T O D O S}

O estudo é um ensaio clínico do tipo antes e depois, segundo Fletcher et al. ${ }^{16}$, e incluiu 25 mulheres com diagnóstico anátomo-patológico de câncer de mama, em Estadiamento Clínico (EC) I e II, submetidas à cirurgia e ao tratamento quimioterápico adjuvante, recrutadas no Departamento de Mastologia do Hospital AC Camargo (São Paulo, Brasil) por amostragem não-probabilística consecutiva, no período de outubro de 2005 a abril de 2006. Durante o período de desenvolvimento do estudo, 377 mulheres tiveram diagnóstico de câncer de mama operável e, dessas 35 tiveram o diagnóstico anátomo-patológico de câncer de mama em estadiamento clínico I e II, seguido de tratamento quimioterápico adjuvante exclusivo. Todas as pacientes ( $n=35$ ) foram convi- 
dadas a participar do estudo, mas somente 25 $(71,4 \%)$ aceitaram e concluíram todas as etapas do protocolo de pesquisa. Desse modo, a amostra inclusa no estudo é representativa para a rotina de atendimento do referido hospital no referido período. Foram excluídas do estudo, pacientes submetidas a tratamento quimioterápico prévio; submetidas à cirurgia nos últimos 12 meses, além da cirurgia da mama; submetidas à radioterapia associada ou a tratamento radioterápico prévio; à presença de neoplasia associada; à presença de afecção crônica transmissível (HIV) ou não transmissível (dislipidemias, diabetes, insuficiência hepática ou renal); as que apresentavam problemas neurológicos ou psiquiátricos; que tinham alguma disfunção gastrointestinal prévia ao tratamento (disfagia, colite, gastrite, úlcera duodenal e gástrica, hérnia de hiato e esofagite) ou que eram alcoolistas.

A coleta de dados foi iniciada após aprovação pelos Comitês de Ética da Faculdade de Saúde Pública (USP) (nº 140/05) e do Hospital AC Camargo da Fundação Antônio Prudente ( $n^{\circ}$ 687/05) e consentimento das pacientes, após assinatura do Termo de Consentimento Livre e Esclarecido. A obtenção dos dados foi realizada em dois momentos: T0 - após o diagnóstico e a cirurgia e antes do primeiro ciclo de quimioterapia e T1 - ao término do último ciclo de quimioterapia. O intervalo médio entre os dois momentos foi de 5 meses (3 - 7 meses).

Os dados de perfil socioeconômico e da história clínica das pacientes foram obtidos por meio de pesquisa nos prontuários no Serviço de Arquivos Médicos e Estatísticos (SAME) e por entrevista direta com as pacientes.

Os parâmetros antropométricos avaliados foram: peso atual, altura e composição corporal. O peso atual foi obtido no momento da avaliação, medido em balança digital Contro| ${ }^{\circledR}$ (Plenna, São Paulo, Brasil), com capacidade de $150 \mathrm{~kg}$ e precisão de $100 \mathrm{~g}$. Foi considerada modificação de peso, o aumento ou a perda no T1 superior a $5 \%$ do peso obtido no baseline (TO), segundo recomendação de Harvie et al. ${ }^{17}$. A altura foi medida utilizando o estadiômetro do modelo AlturaExata ${ }^{\circledR}$ (TBW, São Paulo, Brasil), com limite de $2,10 \mathrm{~m}$ e precisão de 1,0mm. O protocolo de obtenção das medidas de peso e altura seguiu a metodologia descrita por Duarte \& Castellani ${ }^{18}$. Todas as medidas foram coletadas em triplicata.

Para classificação das pacientes de acordo com o estado nutricional foi utilizado o Índice de Massa Corporal (IMC), segundo a Organização Mundial de Saúde ${ }^{19}$.

O consumo e as aversões alimentares foram avaliados utilizando-se o método recordatório de 24h (R24h) e a escala Food Action (FACT) ${ }^{20}$, respectivamente. Para o R24h, pesquisadores treinados conduziram a entrevista e pediram para que a paciente listasse todos os alimentos consumidos no dia anterior, iniciando pelo primeiro alimento.

Para avaliar o consumo alimentar foram utilizadas as seguintes variáveis: energia, proteínas, carboidratos, lipídios, ácidos graxos saturados, ácidos graxos polinsaturados, ácidos graxos monoinsaturados, colesterol, fibras, vitamina $A$, vitamina $C$, vitamina $E$, folato e zinco. Os resultados obtidos a partir dos recordatórios de 24 horas foram analisados no programa Nutwin ${ }^{\circledR}$, versão $1.5^{21}$.

Na coleta de dados de aversões alimentares foi solicitado à paciente que pensasse em sua relação emocional com os alimentos presentes na lista, no quanto gostava ou não deste alimento, para então escolher a resposta correta. As pacientes tinham 9 opções de respostas, cada uma equivalente a um escore determinado (variando de 0 a 9), sendo o escore de menor valor para a opção "como somente se for forçada" e o escore de maior valor para "como sempre que tenho oportunidade". Dessa forma, escores maiores eram indicadores de preferência por determinado alimento/grupo e escores menores indicavam aversão alimentar. As aversões foram avaliadas nos alimentos e nos grupos de alimentos.

Além dessa variável, foram utilizadas para análise de aversão alimentar as respostas à 
seguinte pergunta: "Há algum alimento de que você não goste e que comê-lo ou pensar nele faça você sentir-se desconfortável?" . Foram consideradas como aversão adquirida somente as respostas para aqueles alimentos/grupos descritos a partir do momento T1 (após o tratamento quimioterápico), e que não foram citados pelas pacientes no T0 (antes).

Para avaliar a qualidade de vida, foi utilizada a escala Functional Assessment Cancer Treatment - Breast (FACT-B) traduzida para o português e validada ${ }^{22}$. As pacientes foram orientadas a responder cada item de acordo com as opções apresentadas no questionário: não, pouco, às vezes, muito e sempre $(0,1,2,3$ e 4 pontos, respectivamente); escolhendo a que mais se aplicava à sua situação atual. Os escores do FACT-B para bem-estar físico (contendo 7 itens), social/familiar e funcional (contendo 7 itens) variavam de 0 - 28; para bem-estar emocional (contendo 6 itens), 0 - 24 e, para preocupações adicionais (contendo 9 itens), de 0 - 36.

Na análise do total de escores em cada aspecto de qualidade de vida, ao valor do escore assinalado pelas pacientes foram atribuídos pesos (zero ou quatro), conforme recomendação do guia FACT-B. A soma de todos os escores indicou o nível de qualidade de vida, ou seja, quanto mais alta a soma de escores, melhor a qualidade de vida do indivíduo.

A coleta dos dados foi realizada por nutricionistas ou estudantes do curso de Nutrição previamente treinados e por meio de entrevista direta. Tanto no T0, quanto no T1, todas as entrevistas foram realizadas em consultórios do Departamento de Mastologia do Hospital AC Camargo, mediante prévio agendamento, visando conferir tranqüilidade e privacidade às pacientes, assim como uma correta coleta de informações.

A organização das variáveis foi realizada com auxílio do programa EPIDATA ${ }^{\circledR}$, versão $3.1^{23}$. A aferição da qualidade dos dados foi realizada a partir de dupla digitação. A análise descritiva da população do estudo e a análise comparativa das variáveis foram realizadas com auxílio dos pro- gramas SPSS ${ }^{\circledR}$, versão $13.0^{24}$, SAS ${ }^{\circledR}$ versão $9.1^{25}$ e MINITAB $^{\circledR}$ versão $14.1^{26}$

O teste de Kolmogorov-Smirnov foi aplicado para verificar a normalidade de variáveis. Variáveis quantitativas com distribuição normal foram comparadas entre dois momentos pareados utilizando o teste $t$ de Student para amostras dependentes. Para as variáveis não-paramétricas foi aplicado o teste de Wilcoxon. Análises de correlações lineares intra e entre os tempos (T0 e T1) foram efetuadas utilizando-se o coeficiente de correlação linear de Pearson. Todas as probabilidades de significância ( $p$ ) apresentadas foram do tipo bilateral e foram considerados estatisticamente significantes os valores de $p<0,05$.

\section{RESULTADOS}

A descrição do perfil socioeconômico, cultural e clínico das pacientes encontra-se na Tabela 1. As pacientes apresentaram idade média de 46 (Desvio-Padrão - DP= 9 anos). A maioria (64\%) era casada e se classificou como da raça branca (80\%). A renda familiar foi superior a 7 Salários-Mínimos (SM) em $88 \%$ das pacientes e $64 \%$ delas tinham curso superior completo. Todos esses dados foram coletados apenas na primeira entrevista (T0).

Segundo o perfil clínico, 96\% das pacientes apresentavam neoplasia localizada nos ductos mamários (carcinoma ductal) e 68\% das pacientes receberam diagnóstico de EC tipo II. Dentre as pacientes, $56 \%$ estavam na pré-menopausa e $44 \%$ pós-menopausadas, no momento do diagnóstico. Durante o desenvolvimento deste estudo as pacientes receberam quimioterapia intravenosa, com ciclos de 21 dias. A maioria das pacientes recebeu protocolo quimioterápico antracíclico e ciclofosamida - AC (60\%). A náusea, dentre os efeitos colaterais ao tratamento, foi o de maior freqüência entre as pacientes, atingindo mais de $80 \%$ da população estudada (Tabela 1). A variação de peso $(p<0,001)$ e o IMC $(p<0,001)$ entre os momentos T0 e T1 foi significante, embora esse perfil não tenha sido reflexo de modi- 
800 | S.M.M.L. VERDE et al.

Tabela 1. Caracterização das pacientes inclusas no estudo, de acordo com os perfis socioeconômico e cultural. Hospital AC Camargo. São Paulo (SP), 2005-2006.

\begin{tabular}{|c|c|c|c|c|c|c|c|c|c|c|c|}
\hline \multicolumn{12}{|c|}{ Variáveis } \\
\hline Idade (anos) & $n$ & $\%$ & Escolaridade & $n$ & $\%$ & Quimioterápico & $\mathrm{n}$ & $\%$ & Efeitos colaterais & $n$ & $\%$ \\
\hline $11 \dashv 30$ & 2 & 8 & Fundamental completo & 1 & 4 & $A C$ & 15 & 60 & Constipação & 15 & 60 \\
\hline $31 \dashv 50$ & 13 & 56 & Médio incompleto & 2 & 8 & $A C+T$ & 4 & 16 & Diarréia & 13 & 52 \\
\hline \multirow[t]{3}{*}{$51 \dashv 70$} & 9 & 36 & Médio completo & 3 & 12 & TAC & 3 & 12 & Vômitos & 13 & 52 \\
\hline & & & Superior incompleto & 3 & 12 & FEC & 2 & 8 & Náusea & 22 & 88 \\
\hline & & & Superior completo & 14 & 64 & CMF & 1 & 4 & Lesões orais & 14 & 56 \\
\hline Estado civil & $n$ & $\%$ & Renda familiar (SM) & $\mathrm{n}$ & $\%$ & Etnia & $n$ & $\%$ & Estado de menopausa & $\mathrm{n}$ & $\%$ \\
\hline Casada & 16 & 64 & $2-16$ & 3 & 12 & Branco & 20 & 80 & Pré & 14 & 56 \\
\hline Solteira & 5 & 20 & $7 \dashv 10$ & 12 & 52 & Amarelo & 4 & 16 & Pós & 11 & 44 \\
\hline Separada & 4 & 16 & $>10$ & 9 & 36 & Negro & 1 & 4 & & & \\
\hline Localização & & & & & & & & & Estadiamento clínico & & \\
\hline Ca lobular & 1 & 4 & & & & & & & $\mathrm{EC} \mathrm{I}$ & 8 & 32 \\
\hline Ca ductal & 24 & 96 & & & & & & & EC II & 17 & 68 \\
\hline
\end{tabular}

AC: antracíclico e ciclofosfamida; AC + T: antracíclico, ciclofosfamida e tamoxifeno; TAC: docetaxel, doxorrubicina e ciclofosfamida; FEC: 5-fluorouracil, epirrubicina e ciclofosfamida; CMF: ciclofosfamida, metotrexate e 5-fluoruracil; SM: salário-mínimo.

ficações significantes nos percentuais de massa gorda $(p=0,41)$ e de massa magra $(p=0,78)$.

Quando avaliados o consumo de energia $(p=0,68)$, carboidratos $(p=0,46)$, proteínas $(p=0,55)$, lipídios $(p=0,69)$, colesterol $(p=0,79)$, ácidos graxos saturados $(p=0,33)$, ácidos graxos monoinsaturados $(p=0,20)$, ácidos graxos poliinsaturados $(p=0,90)$, fibras $(p=0,18)$, zinco $(p=0,74)$, vitamina $C(p=0,17)$, vitamina $E(p=0,79)$, folato $(p=0,31)$ e vitamina $A(p=0,52)$ não foram verificadas diferenças significantes entre as médias dos dois tempos (T1-TO). O consumo de alimentos (sopas, macarronada/lasanha, peixe frito etc.) também não mostrou diferenças significantes entre os momentos T0 e T1. Entretanto, quando se realizou a análise entre os grupos de alimentos (sopas e massas, carnes e peixes, arroz e tubérculos etc.), verificou-se que houve redução significante no consumo do grupo de Frutas e Sucos $(p=0,03)$, após o tratamento quimioterápico.

A avaliação das aversões alimentares dessas pacientes mostrou diferenças significantes entre os dois momentos para determinados alimentos, conforme apresentado na Tabela 2.
Para o café preto, a contagem de escores mostrou-se menor no momento T1; para os demais alimentos, os valores dos escores foram maiores após o tratamento quimioterápico.

Quando foram avaliados os escores de aversão para grupos de alimentos (Tabela 3), observou-se redução significante nos escores do grupo das Bebidas (escore $=-5,04, D P=7,39$; $p=0,00$ ), que engloba bebidas alcoólicas, chá, café preto e adoçante, entre os tempos T0 e T1. Os demais grupos de alimentos avaliados permaneceram estatisticamente semelhantes entre os dois tempos.

Para a pergunta "há algum alimento de que você não goste e que comê-lo ou pensar nele faça você sentir-se desconfortável?", verificamos que, no momento $\mathrm{T} 1,52 \%$ das pacientes citaram pelo menos um alimento ou grupo, indicando que desenvolveram aversões alimentares subseqüentes ao tratamento. Entre os mais citados estão os alimentos gordurosos (38\%), seguidos pelo grupo de leite e derivados (23\%). Foram citados ainda café preto $(15 \%)$, chá $(15 \%)$, chocolate $(7 \%)$ e carne vermelha $(7 \%)$. 
Tabela 2. Escores de aversões alimentares de mulheres inclusas no estudo, obtidos nos momentos To e T1. Hospital AC Camargo, São Paulo (SP), 2005-2006.

\begin{tabular}{|c|c|c|c|c|}
\hline Variáveis & Tempo & Média & Desvio-padrão & $p$ \\
\hline \multirow[t]{2}{*}{ Carne bovina (cozida, assada, grelhada ou churrasco) } & T0 & 5,6 & 2,7 & \multirow{2}{*}{0,04} \\
\hline & $\mathrm{T} 1$ & 6,2 & 2,3 & \\
\hline \multirow[t]{2}{*}{ Arroz branco } & T0 & 7,3 & 1,9 & \multirow{2}{*}{0,02} \\
\hline & $\mathrm{T} 1$ & 7,9 & 1,7 & \\
\hline \multirow[t]{2}{*}{ Laranja e mexerica } & T0 & 7,0 & 1,7 & \multirow{2}{*}{0,04} \\
\hline & $\mathrm{T} 1$ & 7,6 & 1,7 & \\
\hline \multirow[t]{2}{*}{ Mamão } & T0 & 7,2 & 1,9 & \multirow{2}{*}{0,04} \\
\hline & $\mathrm{T} 1$ & 7,7 & 1,8 & \\
\hline \multirow[t]{2}{*}{ Biscoito salgado/biscoito doce sem recheio } & T0 & 5,4 & 2,3 & \multirow{2}{*}{0,03} \\
\hline & $\mathrm{T} 1$ & 6,6 & 2,2 & \\
\hline \multirow{2}{*}{ Café preto } & T0 & 7,4 & 1,9 & \multirow{2}{*}{0,01} \\
\hline & $\mathrm{T} 1$ & 5,8 & 3,0 & \\
\hline \multirow[t]{2}{*}{ Sorvete } & T0 & 5,7 & 1,8 & \multirow{2}{*}{0,00} \\
\hline & $\mathrm{T} 1$ & 6,4 & 1,9 & \\
\hline
\end{tabular}

$\mathrm{n}=25$. Diferenças entre os tempos foram estabelecidas pelo teste $t$ de Student e Wilcoxon, com nível de significância de $p<0,05$. T0: tempo basal; T1: após o tratamento quimioterápico.

Tabela 3. Escores de aversões alimentares de mulheres inclusas no estudo, segundo os grupos de alimentos, nos momentos T0 e T1. Hospital AC Camargo, São Paulo (SP), 2005-2006.

\begin{tabular}{|c|c|c|c|c|}
\hline Variáveis & Tempo & Média & Desvio-padrão & $p$ \\
\hline \multirow[t]{2}{*}{ Sopas e massas } & T0 & 19,8 & 4,2 & \multirow{2}{*}{0,92} \\
\hline & T1 & 19,8 & 2,8 & \\
\hline \multirow[t]{2}{*}{ Carnes e peixes } & T0 & 49,5 & 12,2 & \multirow{2}{*}{0,49} \\
\hline & T1 & 50,4 & 12,9 & \\
\hline \multirow[t]{2}{*}{ Leguminosas e ovo } & T0 & 20,4 & 3,3 & \multirow{2}{*}{0,39} \\
\hline & T1 & 19,8 & 3,4 & \\
\hline \multirow[t]{2}{*}{ Arroz e tubérculos } & T0 & 40,1 & 11,2 & \multirow{2}{*}{0,05} \\
\hline & T1 & 43,7 & 11,5 & \\
\hline \multirow[t]{2}{*}{ Leite/derivados e cereais } & T0 & 35,5 & 11,3 & \multirow{2}{*}{0,57} \\
\hline & T1 & 36,6 & 11,3 & \\
\hline \multirow[t]{2}{*}{ Vegetais } & T0 & 44,3 & 7,0 & \multirow{2}{*}{0,86} \\
\hline & T1 & 43,6 & 8,3 & \\
\hline \multirow[t]{2}{*}{ Molhos } & T0 & 15,8 & 3,8 & \multirow{2}{*}{0,07} \\
\hline & T1 & 16,3 & 4,9 & \\
\hline \multirow{2}{*}{ Frutas e sucos } & T0 & 68,6 & 11,3 & \multirow{2}{*}{0,29} \\
\hline & T1 & 70,6 & 11,9 & \\
\hline \multirow[t]{2}{*}{ Pães e biscoitos } & T0 & 29,2 & 6,5 & \multirow{2}{*}{0,48} \\
\hline & T1 & 30,0 & 5,9 & \\
\hline \multirow[t]{2}{*}{ Bebidas } & T0 & 23,8 & 6,5 & \multirow{2}{*}{$0,00^{*}$} \\
\hline & T1 & 18,7 & 6,8 & \\
\hline \multirow[t]{2}{*}{ Doces, sobremesas e aperitivos } & T0 & 29,5 & 6,8 & \multirow{2}{*}{0,97} \\
\hline & T1 & 29,5 & 6,8 & \\
\hline \multirow[t]{2}{*}{ Total de escores } & T0 & 376,5 & 46,5 & \multirow{2}{*}{0,73} \\
\hline & T1 & 378,9 & 49,7 & \\
\hline
\end{tabular}

$n=25$. Diferenças entre os tempos foram estabelecidas pelo teste $t$ de Student e Wilcoxon, com nível de significância de $p<0,05$. T0: tempo basal; T1: após o tratamento quimioterápico. 
802 S.M.M.L. VERDE et al.

Quanto à qualidade de vida, a Tabela 4 descreve as variáveis analisadas com seus respectivos escores antes e após o tratamento quimioterápico. Houve diferença significante para o aspecto de bem-estar físico (escore=-3,47; $p=0,00$ ), que engloba questões associadas à presença de dor, capacidade de atender às necessidades da família, sensação de fraqueza e náuseas e incômodo pelos efeitos colaterais do tratamento quimioterápico. Para as demais variáveis não houve alterações significantes entre os tempos T0 e T1.

Tabela 4. Escores de qualidade de vida de mulheres inclusas no estudo, relacionados com bem-estar físico, bem-estar social/familiar, bem-estar emocional, bem-estar funcional e com preocupações adicionais. Hospital AC Camargo, São Paulo (SP), 2005-2006.

\begin{tabular}{|c|c|c|c|c|}
\hline Variáveis & Tempo & Média & Desvio-padrão & $p$ \\
\hline \multirow[t]{2}{*}{ Bem-estar físico } & T0 & 22,2 & 4,9 & \multirow{2}{*}{0,00} \\
\hline & $\mathrm{T} 1$ & 18,8 & 5,0 & \\
\hline \multirow[t]{2}{*}{ Bem-estar social/familiar } & T0 & 16,1 & 4,1 & \multirow{2}{*}{0,28} \\
\hline & $\mathrm{T} 1$ & 16,9 & 2,4 & \\
\hline \multirow[t]{2}{*}{ Bem-estar emocional } & T0 & 16,5 & 4,9 & \multirow{2}{*}{0,52} \\
\hline & $\mathrm{T} 1$ & 16,9 & 4,0 & \\
\hline \multirow[t]{2}{*}{ Bem-estar funcional } & T0 & 17,2 & 5,8 & \multirow{2}{*}{0,56} \\
\hline & $\mathrm{T} 1$ & 18,1 & 6,3 & \\
\hline \multirow[t]{2}{*}{ Preocupações adicionais } & TO & 21,4 & 5,1 & \multirow{2}{*}{0,11} \\
\hline & $\mathrm{T} 1$ & 19,5 & 5,1 & \\
\hline \multirow[t]{2}{*}{ Total de escores } & TO & 91,8 & 18,4 & \multirow{2}{*}{0,43} \\
\hline & $\mathrm{T} 1$ & 90,1 & 16,8 & \\
\hline
\end{tabular}

$\mathrm{n}=25$. Diferenças entre os tempos foram estabelecidas pelo teste $t$ de Student e Wilcoxon, com nível de significância de $p<0,05$. T0: tempo basal; T1: após o tratamento quimioterápico.

Tabela 5. Correlação entre comportamento alimentar e qualidade de vida de mulheres inclusas no estudo, no momento T1. Hospital AC Camargo. São Paulo (SP), 2005-2006.

\begin{tabular}{|c|c|c|c|}
\hline Variável & Variável de correlação & r & $p$ \\
\hline & Nutrientes & & \\
\hline Carboidratos (g) & Bem-estar funcional & $-0,434$ & 0,03 \\
\hline \multirow[t]{2}{*}{ Proteínas (g) } & Bem-estar emocional & 0,472 & 0,01 \\
\hline & Grupos de alimentos & & \\
\hline Leite/derivados e cereais & Bem-estar social & $-0,309$ & 0,06 \\
\hline Vegetais & Bem-estar funcional & $-0,440$ & 0,01 \\
\hline \multirow[t]{2}{*}{ Frutas e sucos } & Bem-estar social & 0,368 & 0,03 \\
\hline & Bem-estar funcional & $-0,442$ & 0,01 \\
\hline Pães e biscoitos & Questões de interesse adicional & $-0,356$ & 0,04 \\
\hline \multirow[t]{2}{*}{ Bebidas } & Bem-estar funcional & $-0,380$ & 0,03 \\
\hline & Grupos de aversões alimentares & & \\
\hline \multirow[t]{2}{*}{ Arroz e tubérculos } & Bem-estar físico & 0,514 & 0,00 \\
\hline & Bem-estar funcional & 0,556 & 0,00 \\
\hline \multirow[t]{2}{*}{ Leite e derivados } & Bem-estar emocional & 0,468 & 0,01 \\
\hline & Bem-estar funcional & 0,552 & 0,00 \\
\hline Sopas e massas & Bem-estar funcional & 0,534 & 0,00 \\
\hline Carnes e peixes & Bem-estar funcional & 0,415 & 0,04 \\
\hline Vegetais & Preocupações adicionais & 0,422 & 0,03 \\
\hline
\end{tabular}

(r) Coeficiente de correlação de Pearson. O nível de significância adotado de $p<0,05$. T1: após o tratamento quimioterápico. 
Foram verificadas as possíveis correlações entre o comportamento alimentar e a qualidade de vida, sendo os resultados descritos na Tabela 5. De acordo com esses resultados, observaram-se correlações significantes entre essas variáveis no momento $\mathrm{T} 1$, as quais não existiam antes do tratamento quimioterápico (T0).

\section{I S C U S S Ã O}

O aumento do número de sobreviventes ao câncer de mama reforça a necessidade de investigar o impacto a longo prazo da própria doença e dos efeitos colaterais dos tratamentos antineoplásicos disponíveis ${ }^{27}$. Nesse contexto, o aumento da sobrevida e a diminuição do risco de recidivas são confrontados com a experiência de efeitos como náusea, vômitos e alterações no padrão alimentar, que podem comprometer a qualidade de vida das pacientes ${ }^{28}$.

Manter o estado nutricional de eutrofia é reconhecido como parte fundamental do tratamento do câncer, e os profissionais envolvidos no cuidado do paciente devem estar conscientes dos fatores fisiopatológicos e psicológicos que interferem na aceitação dos alimentos ${ }^{12}$. De acordo com os resultados antropométricos verificou-se que o aumento no peso esteve associado ao ganho significante de massa gorda e à redução da massa magra. Esses resultados reforçam o impacto do comportamento alimentar após o diagnóstico e o tratamento quimioterápico.

As escolhas alimentares de pacientes com neoplasia mamária podem ser alteradas quando, em função dos efeitos colaterais resultantes do tratamento quimioterápico, ocorre rejeição a determinados alimentos ou bebidas ${ }^{13}$. Este fenômeno é conhecido como aversão alimentar adquirida.

A causa das mudanças nos padrões alimentares dessas pacientes ainda não está claramente definida, podendo ser devido às modificações sensoriais do paladar, com alterações no limiar para determinados sabores ${ }^{29,10}$. Esses fatores podem influenciar diretamente a ingestão alimen- tar dessas mulheres e contribuir para a alteração nos hábitos alimentares previamente estabelecidos.

Os escores de preferências alimentares antes (T0) e após (T1) o tratamento quimioterápico mostraram redução significante para o consumo de café preto $(p=0,011)$ e do grupo de bebidas $(p=0,02)$, indicando aversão após o tratamento quimioterápico. Previamente tem sido mostrado que mulheres com câncer mama em EC iniciais apresentam menores níveis de aversões alimentares adquiridas $(p=0,014)$ que pacientes com doença avançada ${ }^{11}$. Aversões alimentares adquiridas diminuem com a continuidade do tratamento $^{30}$. É provável que a classificação destas pacientes dentro dos EC I e II seja determinante para que a redução nos escores de aversão alimentar entre os dois momentos (TO-T1) tenha sido significante somente para o café e para o grupo das bebidas. Considera-se ainda que a falta de aversões observadas após o último ciclo de quimioterapia, para a maioria dos alimentos, possa indicar que tais modificações nas escolhas alimentares, potencialmente ocorridas durante o tratamento, foram transitórias.

No que se refere à pergunta "Há algum alimento de que você não goste e que comê-lo ou pensar nele faça você sentir-se desconfortável?", este estudo verificou a presença de aversões a, pelo menos, um item alimentar em $52 \%$ das pacientes após a quimioterapia, pois no T0 esses alimentos não foram descritos quando as pacientes foram questionadas para essa mesma pergunta. Em estudo com 76 pacientes com câncer foi verificado que mais de $50 \%$ da população desenvolveu algum tipo de aversão alimentar ${ }^{11}$. Até $82 \%$ das mulheres com câncer de mama em quimioterapia podem apresentar quadro de aversão alimentar adquirida ${ }^{31}$.

Do total de pacientes que relataram a presença de aversão após a quimioterapia (T1), 38\% delas associaram alimentos gordurosos com sensação de incômodo e mal-estar. Esses alimentos são conhecidos como potentes estimuladores de secreção ácido-gástrica, causando o mal-estar característico do refluxo gastro-esofágico ${ }^{13}$. O café 
804 | S.M.M.L. VERDE et al.

preto foi citado por $15 \%$ das pacientes como preparação que trazia sensação de desconforto. O chá e o chocolate foram alimentos igualmente mencionados por $15 \%$ e $7 \%$ das pacientes, respectivamente, desse estudo como rejeitados após a terapia antineoplásica adjuvante. Café, chá e chocolate têm sido descritos como alimentos comuns no registro de aversão, assim como frutas cítricas e carne vermelha ${ }^{31}$. A tendência a evitar carnes, chá e chocolate está associada ao aumento da percepção do sabor amargo e, portanto, aos efeitos colaterais da quimioterapia, sobretudo a alterações sensoriais do paladar ${ }^{12}$.

Neste estudo, o consumo de carne vermelha só foi referido como relacionado à sensação de desconforto por 7\% das pacientes. Entre os alimentos do grupo das carnes, constatou-se aumento significante no escore de aversão para carne bovina (cozida, assada, grelhada ou churras$c 0), p=0,04$, indicando preferência por esse item alimentar. Esse percentual é inferior ao encontrado em outro estudo ${ }^{13}$, no qual $26,4 \%$ dos pacientes com câncer em tratamento quimioterápico apresentaram aversão à carne. Nesse estudo, o próprio autor reconhece que a incidência de aversões a esse alimento é desproporcional ao seu consumo, evidenciando a diferença entre o querer e o fazer.

Semelhantemente ao que aconteceu com alguns tipos de carnes, destacou-se um aumento significante na preferência por determinadas frutas (laranja, mamão e mexerica), $p=0,04$, o que se opõe aos achados de que frutas são comumente relatadas como alimentos rejeitados ${ }^{31}$. Essas observações revelam a complexidade do monitoramento do comportamento alimentar, e reforçam a idéia de que as escolhas alimentares podem ser orientadas pela busca da satisfação do paladar, pelo que se deseja comer ou pelo que se deve comer, ou seja, pelo hedonismo ou pela disciplina ${ }^{32}$.

É necessário, portanto, compreender o processo de ingestão do ponto de vista psicológico e sociocultural, e conhecer as atitudes e crenças, além dos fatores fisiopatológicos que influenciam esse processo de decisão ${ }^{33}$.
O alto nível de escolaridade das pacientes envolvidas no presente estudo pode ser destacado como fator que contribui para a tentativa de superação das aversões adquiridas durante o tratamento, uma vez que estar consciente da importância de uma alimentação saudável pode levá-las a consumir uma maior quantidade de vitaminas antioxidantes, por exemplo, resultando no aumento das preferências por laranja, mexerica e mamão. Adicionalmente aos aspectos destacados acima, mais recentemente foi demonstrada a eficácia de consumir alimentos azedos (como as frutas cítricas), a fim de amenizar as alterações do paladar?.

Ainda com relação aos escores de preferências alimentares, observou-se que houve aumento significante na preferência por sorvete $(p<0,0001)$. Neste estudo mais de $50 \%$ das pacientes referiram presença de mucosite e, portanto, acredita-se que o aumento no escore de preferência por sorvete possa ser explicado pela tentativa das pacientes em reduzir os sintomas associados a essa inflamação. Essas observações reforçam a afirmação de que a preferência pelo consumo de alimentos gelados pode ser uma estratégia bastante eficaz na tentativa de amenizar os efeitos colaterais do tratamento quimioterápico sobre as alterações referentes ao paladar ${ }^{7}$.

Foi verificado que pacientes com câncer de mama apresentam aversão alimentar pós-quimioterapia para produtos lácteos ${ }^{34}$. No atual estudo pôde-se observar que apesar de não ter havido modificação significante no escore de preferências para esse grupo de alimentos, $23 \%$ das pacientes citaram leite e derivados como sendo associado ao incômodo no T1. Ao contrário, em estudo realizado com pacientes em quimioterapia, foi observado que, na tentativa de amenizar sintomas como náuseas e vômitos a partir dos próprios conhecimentos, alguns deles recorriam ao consumo do leite ${ }^{35}$. Por outro lado, nesse mesmo estudo, pacientes com câncer de mama referiram consumir biscoitos sem recheio como forma de aliviar efeitos colaterais, dado esse em conformidade com os resultados do presente estudo, 
segundo os quais biscoito salgado/biscoito doce sem recheio $(p=0,03)$ foram alimentos que apresentaram aumento significante no escore de preferências alimentares após o tratamento quimioterápico, o que pode ser justificado pela tentativa individual de se adaptar ao tratamento vivenciado.

Pacientes que sofrem alterações na percepção do paladar são mais suscetíveis a reportar depressão do que aqueles que não apresentam esse sintoma ${ }^{12,36}$. Quarenta por cento dos pacientes oncológicos relataram ter suas vidas afetadas por alterações no sentido do paladar, relataram também o sentimento de depressão ${ }^{36}$. Devido ao fato de os alimentos desempenharam um papel essencial nas atividades sociais, a perda do sabor pode levar também desinteresse e falta de prazer em experiências que envolvam o ato de comer e, inclusive, em um afastamento do contato social, de uma forma geral ${ }^{12,36}$. Neste estudo este achado foi comprovado pelas correlações entre os escores de preferências alimentares e diferentes aspectos de qualidade de vida. Entende-se, portanto que aspectos alimentares, sejam de caráter nutricional ou social, têm forte impacto na resposta ao tratamento antineoplásico e também na qualidade de vida dos pacientes oncológicos.

Além dos aspectos citados acima, é importante destacar as limitações inerentes às ferramentas de inquérito alimentar e, especificamente, do R24h, pois é sabido que os perfis socioeconômico, cultural e clínico influenciam fortemente a ingestão alimentar do paciente. Associada a essas variáveis, destacam-se vários fatores que afetam o processo cognitivo de lembrar e descrever os alimentos, mesmo aqueles consumidos 24 horas antes. Desses, o sexo, a idade e o nível educacional são considerados os mais importantes. Neste estudo, a alta freqüência de mulheres com elevado nível educacional e o relato de que elas executavam atividades domésticas reforçam a qualidade das informações obtidas sobre o consumo alimentar destas pacientes. Embora o R24h, quando comparado aos questionários de freqüência alimentar (QFA), possa não ser a ferramenta mais adequada para avaliar o consumo de micronutrientes, o R24h mostrou-se adequado aos objetivos deste estudo. Portanto, não há o melhor método de consumo, mas sim o mais adequado a uma situação específica ${ }^{37}$, pois a verdadeira ingestão de alimentos e nutrientes constitui uma variável latente, cujos resultados não podem ser considerados livres de erros ${ }^{38}$.

Conclui-se que os resultados apresentados mostram que o comportamento alimentar e a qualidade de vida são interrelacionáveis e são mutuamente influenciados pelo tratamento quimioterápico. Nesse sentido, pesquisas com ênfase no comportamento alimentar devem ser estimuladas visando confirmar os resultados encontrados no presente estudo.

\section{COLABORADORES}

S.M.M.L. VERDE participou ativamente das etapas de coleta, tabulação, análise e discussão dos resultados. B.M.O. SÃO PEDRO participou da avaliação do comportamento alimentar (consumo e aversão), pela aplicação dos instrumentos específicos. M. MOURÃO NETTO participou da seleção das pacientes e da discussão dos resultados no que se refere aos aspectos clínicos. N.R.T. DAMASCENO coordenou a pesquisa e delineou o estudo; participou da interpretação e da discussão dos resultados.

\section{REFERÊ N CIAS}

1. Shapiro SL, Lopez AM, Schwartz GE, Bootzin R, Figueredo AJ, Braden CJ, et al. Quality of life and breast cancer: relationship to psychosocial variables. J Clin Psychol. 2001; 57(4):501-19. doi:10.1002/jclp.1026.

2. Global Cancer Statistics [homepage na internet]. Paris: International Agency for Research on Cancer. [cited 2007 Jan 21]. Available from: <http://www. iarc.fr>.

3. Rock $C L$, Demark-Wahnefried W. Nutrition and survival after the diagnosis of breast cancer: a review of the evidence. J Clin Oncol. 2002; 20(15): 3302-16. doi:10.1200/JCO.2002.03.008.

4. Stull VB, Snyder DC, Demark-Wahnefried W. Lifestyle interventions in cancer survivors: designing programs that meet the needs of this 
806 | S.M.M.L. VERDE et al.

vulnerable and growing population. J Nutr. 2007; 137(Suppl. 1): 243s-8s.

5. Instituto Nacional do Câncer. Estimativa 2008: incidência de câncer no Brasil. Rio de Janeiro: INCA; 2007.

6. Pinho VFS, Coutinho ESF. Risk factors for breast cancer: a systematic review of studies with female samples among the general population in Brazil. Cad Saúde Pública. 2005; 21(2):351-60. doi: 10.1590/S0102-311X2005000200002.

7. Williams SA, Schreier AM. The effect of education in managing side effects in women receiving chemotherapy for treatment of breast cancer. Oncol Nurs Forum. 2004; 31(1):16-23. doi: 10.1188/04.ONF.E16-E23.

8. Comeau TB, Epstein JB, Migas C. Taste and smell dysfunction in patients receiving chemotherapy: a review of current knowledge. Support Care Cancer. 2001; 9(8):575-80. doi:10.1188/04.ONF.E16-E23.

9. Epstein JB, Phillips N, Parry J, Epstein MS, Nevill T, Stevenson-Moore P. Quality of life, taste, olfactory and oral function following high-dose chemotherapy and allogeneic hematopoietic cell transplantation. Bone Marrow Transplant. 2002; 30(11):785-92. doi:10.1038/sj.bmt.1703716.

10. Berteretche MV, Dalix AM, d'Ornaro AM, Bellisle F, Khayat D, Faurion A. Decreased taste sensitivity in cancer patients under chemotherapy. Support Care Cancer. 2004; 12(8):571-6. doi:10.1007/s00520004-0589-2.

11. Mattes RD, Arnold C, Boraas M. Learned food aversion among cancer chemotherapy patients: incidence, nature, and clinical implications. Cancer. 1987; 60(10):2576-80. doi:10.1002/1097-0142 (19871115)60:10.

12. Ravasco P. Aspects of taste and compliance in patients with cancer. Eur J Oncol Nurs. 2005; 9(S2): 84-91. doi:10.1016/j.ejon.2005.09.003.

13. Mattes RD, Curran Jr WJ, Alavi J, Powlis W, Whittington R. Clinical implications of learned food aversions in patients with cancer treated with chemotherapy or radiation therapy. Cancer. 1992; 70(1):192-200. doi:10.1002/1097-0142(19920701) 70:1.

14. Arora NK, Gustafson DH, Hawkins RP, McTavish F, Cella DF, Pingree $S$, et al. Impact of surgery and chemotherapy on the quality of life of younger women with breast carcinoma: a prospective study. Cancer. 2001; 92(5):1288-98.

15. Kuroi K, Shimozuma K, Ohsumi S, Imai H, Ono M. Current status of health outcome assessment of medical treatment in breast cancer. Breast Cancer. 2007; 14(1):74-80. doi:10.2325/jbcs.14.74.
16. Fletcher RH, Fletcher SW, Wagner EH. Epidemiologia clínica: elementos essenciais. 3a. ed. Porto Alegre: Artes Médicas; 1996.

17. Harvie M, Howell A, Vierkant RA, Kumar N, Cerhan $J R$, Kelemen LE, et al. Association of gain and loss of weight before and after menopause with risk of postmenopausal breast cancer in the lowa women's health study. Cancer Epidemiol Biomarkers Prev. 2005. 14(3):656-61. doi: 10.1158/1055-996 5.EPI-04-0001.

18. Duarte AC, Castellani FR. Semiologia nutricional. Rio de Janeiro: Axcel Books do Brasil; 2002.

19. World Health Organization. The problem of overweight and obesity. In: Obesity: preventing and managing the global epidemic. Geneva: World Health Organization; 2000. WHO Technical Report Series, 897.

20. Schutz HG. A food action rating scale for measuring food acceptance. J Food Sci. 1965; 30:365-74. doi: 10.1111/j.1365-2621.1965.tb00316.x.

21. NutWin: programa de apoio à nutrição [CD-Rom]. Versão 1.5. São Paulo: Unifesp; 2002.

22. Brady MJ, Cella DF, Mo F, Bonome AE, Tulsky DS, Lloyd SR, et al. Reliability and validity of the functional assessment of cancer therapy-breast quality-of-life instrument. J Clin Oncol. 1997; 15(3):974-86.

23. Epidata, version 3.1 [software internet]. Denmark: Epidata Association; 2005 [cited 2005 Sept]. Available from: <http://www.epidata.com.br>.

24. Statistical Package for the Social Science [CD-Rom]. Version 13.0. Chicago: Incorporation, 2000.

25. SAS for DOS [CD-Rom]. Version 9.1.3. Cary (NC): SAS Institute, 2006.

26. Minitab Statistical [CD-Rom]. Version 14. State College (PA): Minitab Inc; 2003.

27. Mols F, Vingerhoets AJ, Coebergh JW, van de PollFranse LV. Quality of life among long-term breast cancer survivors: a systematic review. Eur J Cancer. 2005; 41(17):2613-19. doi:10.1016/j.ejca.2005. 05.017.

28. Simes RJ, Coates AS. Patient preferences for adjuvant chemotherapy of early breast cancer: how much benefit is needed? J Natl Cancer Inst Monogr. 2001; (30):146-52.

29. Knibb RC, Smith DM, Booth DA, Armstrong AM, Platts RG, MacDonald A, et al. No unique role for nausea attributed to eating a food in the recalled acquisition of sensory aversion for that food. Appetite. 2001; 36(3):225-34. doi: 10.1006/appe. 2001.0402. 
30. Jacobsen PB, Bovbjerg DH, Schwartz MD, Andrykowski MA, Futterman D, Gilewski T, et al. Formation of food aversion in cancer patients receiving repeated infusions of chemotherapy. Behav Res Ther. 1993; 31(8):738-48. doi:10.1016/ 0005-7967(93)90004-E.

31. Holmes S. Food avoidance in patients undergoing cancer chemotherapy. Support Care Cancer. 1993; 1(6):326-30.

32. Garcia RWD. Práticas e comportamento alimentar no meio urbano: um estudo no centro da cidade de São Paulo. Cad Saúde Pública. 1997; 13(3): 455-467. doi:10.1590/S0102-311X1997000 300021.

33. Viana V. Psicologia, saúde e nutrição: contributo para o estudo do comportamento alimentar. Anál Psicol. 2002; 20(4):611-24.

34. McEligot AJ, Rock CL, Sobo EJ, Flatt SW. Food avoidance by women at risk for recurrence of breast cancer. J Cancer Educ. 2000; 15(3):151-5.
35. Asbury N, Walshe A. Involving women with breast cancer in the development of a patient information leaflet for anticipatory nausea and vomiting. Eur J Oncol Nurs. 2005; 9(1):33-43. doi:10.1016/j.ejon. 2004.07.003.

36. Wickham RS, Rehwaldt M, Kefer C, Shott S, Abbas K, Glynn-Tucker E, et al. Taste changes experienced by patients receiving chemotherapy. Oncol Nurs Forum. 1999; 26(4):697-706.

37. Albert US, Koller M, Wagner U, Schultz KD. Survival chances and psychological aspects of quality of life in patients with localized early stage breast cancer. Inflamm Res. 2004; 53(2S):136-41. doi:10.1007/s00011-004-0365-2.

38. Aslani A, Smith RC, Allen BJ, Pavlakis N, Levi JA. Change in body composition during breast cancer chemotherapy with the CMF-regimen. Breast Cancer Res Treat. 1999; 57(3):285-90.

Recebido em: 7/12/2007

Versão final reapresentada em: 21/7/2008 Aprovado em: 27/4/2009 